\title{
Aplicación de la ingeniería didáctica en el aprendizaje del concepto de función
}

\section{Application of didactic engineering in the learning of the concept of function}

\author{
Aplicação de engenharia didática na aprendizagem do conceito de função \\ Ashley Gallo -Silva ${ }^{a}$, Jeriserth Manrique -Jaimes ${ }^{\mathrm{b}}$, Raúl Prada -Núñez ${ }^{\mathrm{c}^{*}}$ \\ Estudiantes del Semillero en Educación Matemática adscrito al Grupo GIPEPP. \\ Orcid: 0000-0002-7630-6212, Orcid: 0000-0002-7263-8180 \\ Maestría en Educación Matemática \\ abc Universidad Francisco de Paula Santander. Cúcuta, Colombia. Orcid: 0000-0001-6145-1786
}

Forma de citar: Gallo, A., Manrique, J., Prada, R., (2017). Aplicación de la ingeniería didáctica en el aprendizaje del concepto de función. Eco matemático 8(1). 43-48

Recibido: agosto 5 de 2016

Aceptado: noviembre 02 de 2016

\section{Palabras clave}

secuencias didácticas, función, representaciones semióticas.

\section{Keywords \\ didactic sequences, function, semiotic representations.}

\begin{abstract}
Resumen: El concepto matemático de función es el que facilita hacer la transición entre la educación básica media y la educación superior, pero a pesar de abordarse en diversos grados desde el colegio, en diversas investigaciones precedidas a esta, se ha identificado problemas en estudiantes en su primer curso de Cálculo al no tener la correcta apropiación del concepto, puesto que este es fundamental para el aprendizaje de los diversos contenidos propios del Cálculo como lo son límite, continuidad, derivada. Debido a éste contexto de dificultad es el que hace necesario la aplicación de nuevas metodologías de investigación que tiendan a favorecer una enseñanza exitosa que propenda por la correcta apropiación del concepto de función. Para lo cual se han propuesto una serie de secuencias didácticas que se encuentran estructuradas bajo las dificultades identificadas en una prueba diagnóstica y en el conocimiento del estado del arte del tema. Las secuencias didácticas se apoyan en la teoría de las representaciones semióticas de Duval, con lo que se pretende que los estudiantes puedan reconocer una función en cualquier tipo de registro de representación y poder articular un registro con otro de manera coherente. La investigación se realiza con estudiantes del programa de Licenciatura en Matemáticas de la UFPS, de los cuales se elige una muestra intencional no probabilística integrada por los estudiantes de II semestre de Licenciatura en Matemáticas.
\end{abstract}

\begin{abstract}
The mathematical concept of function is the one that it facilitates to do the transition between the basic average education and the top education, but in spite of be approaching in diverse degrees from the college, in diverse investigations preceded this one, problems have been identified in students in his first course of Calculation on not having had the correct appropriation of the concept, since this one is fundamental for the learning of the diverse own contents of the Calculation as it it are limit, continuity, derivative. Due to this one context of difficulty is the one that makes necessary the
\end{abstract}

* Autor para correspondencia raulprada@ufps.edu.co

http://dx.doi.org/10.22463/17948231.1474 
application of new methodologies of investigation that tend to favor a successful education that it tends for the correct appropriation of the concept of function. For which they have proposed a series of didactic sequences that are structured under the difficulties identified in a diagnostic test and in the knowledge of the condition of the art of the topic. The didactic sequences rest on the theory of the semiotics representations of Duval, with what there is claimed that the students could recognize a function in any type of record of representation and to be able to articulate a record with other one in a coherent way. The research is carried out with students of the UFPS Mathematics Bachelor program, of which an intentional sample chooses non-probabilistic integrated by students of the second semester of the Bachelor of Mathematics.

\section{Palavras-chave}

seqüências didáticas, função, representações semióticas.
Resumo: O conceito matemático de função é o que facilita a transição entre o ensino básico e o ensino superior, mas, apesar de ser abordado em vários graus da escola, em várias investigações anteriores, os alunos foram identificados em sua primeira Curso de cálculo para não ter a correta apropriação do conceito, pois isso é essencial para aprender os vários conteúdos do cálculo, pois são limite, continuidade, derivativo. Devido a este contexto de dificuldade é o que torna necessária a aplicação de novas metodologias de pesquisa que tendem a favorecer um ensino bem sucedido que propicie a correta apropriação do conceito de função. Para o qual foi proposta uma série de sequências didáticas estruturadas sob as dificuldades identificadas em um teste diagnóstico e no conhecimento do estado da arte do sujeito. As seqüências didáticas baseiam-se na teoria de representações semióticas de Duval, com a qual se pretende que os estudantes reconheçam uma função em qualquer tipo de registro de representação e sejam capazes de articular um registro com outro de maneira coerente. A pesquisa é realizada com alunos do programa de Bacharelado em Matemática da UFPS, a partir do qual é escolhida uma amostra intencional não probabilística, integrada pelos alunos do segundo semestre do Bacharelado em Matemática.

\section{Introducción}

El concepto de función es uno de los más difíciles tanto para su enseñanza como para su aprendizaje y no se puede pretender que un estudiante tenga total apropiación del mismo a partir de una clase expositiva, siendo esta una de las principales problemáticas que se presentan en la educación. Tanto docentes y estudiantes han restringido el concepto a un simple proceso de mecanización, teniendo un conjunto de pasos para resolver ejercicios estandarizados, llevando a cabo una enseñanza y aprendizaje tradicional (Eisenberg, 1991, Hitt, 1998). En la mayor parte de los casos al iniciar el primer curso de Cálculo los estudiantes tienen dificultades en el momento de abordar contenidos como límite o derivada, y esto sucede debido a que no tienen claro el concepto de función (Prada-Núñez, HernándezSuárez \& Ramírez-Leal, 2016). En este orden de ideas con el fin de que los estudiantes tengan total apropiación del concepto de función, siendo este fundamental en el aprendizaje del cálculo se busca implementar las secuencias didácticas bajo la teoría de las representaciones semióticas de Duval y la ingeniería didáctica como método de investigación.

En investigaciones precedidas a esta se han encontrado problemáticas alrededor de este concepto, López y Sosa (2008) en su investigación encuentran dificultades en sus estudiantes de bachiller, como distinguir entre variable e incógnita y el manejo operacional arbitrario de funciones como si fueran ecuaciones. Todas estas problemáticas encontradas a nivel de educación básica media y educación superior fueron contrastadas con la población a trabajar obteniendo que muchas de ellas se presentaban con frecuencia en los docente en formación, por lo 
que se hace necesario elaborar un plan de acción que al intervenir genere cambios en el proceso de enseñanza y aprendizaje del concepto de función. Es por esto que las secuencias didácticas definidas por Tobón (2010) como "conjuntos articulados de actividades de aprendizaje y evaluación que, con la mediación de un docente, buscan el logro de determinadas metas educativas, considerando una serie de recursos." (p.20) se implementan para que por medio de actividades debidamente organizadas se pueda generar un efecto positivo en el proceso de aprendizaje de los estudiantes.

\section{Materiales y métodos}

Para la investigación se adoptó la metodología de investigación denominada Ingeniería Didáctica como marco referencial, donde se realizó un análisis inicial de las dificultades que habitualmente se presentaron alrededor del concepto de función (derivado de la construcción del estado del arte de tema y de investigaciones preliminares que se han desarrollado en la UFPS) que sumado al desarrollo de unas actividades pre-instruccionales que consistieron en el planteamiento y aplicación de un instrumento diagnóstico a fin de identificar los conocimientos previos alrededor del concepto de función en un grupo de docentes en formación. Posteriormente, los investigadores diseñaron una serie de actividades en las que se buscaba que los estudiantes cuestionados estuvieran inmersos en la solución de secuencias didácticas fundamentadas en la articulación de diversos registros de representación semiótica, con el fin de poder analizar los efectos derivados de estas, en el aprendizaje del concepto de función.

En el instrumento diagnóstico requirieron conectar los diferentes sistemas de representación semiótica. Los cuestionamientos incluidos es este instrumento consistieron en la determinación del concepto de par ordenado, coordenadas de un punto, ubicación en el plano cartesiano, estudio de los cuadrantes, reconocimiento de ejes. La aplicación del instrumento fue sin previo aviso y la duración fue de noventa minutos, en donde contestaron sin el uso de fuentes o bibliografía, las valoraciones fueron analizadas bajo la metodología cuantitativa. La investigación se llevó a cabo durante el curso de Cálculo Diferencial al grupo de segundo semestre de Licenciatura en Matemáticas de la Universidad Francisco de Paula Santander en el 2018-A (un total de 25 estudiantes).

\section{Resultados y discusión}

\section{Primer Momento: Prueba inicial}

Para dar inicio a la elaboración de secuencias didácticas se analizó la prueba diagnóstica que consistió en una revisión de conocimientos previos para el desarrollo del aprendizaje del concepto de función, la cual fue aplicada a 25 docentes en formación del segundo semestre del programa de Licenciatura en Matemáticas. La prueba inicia con el reconocimiento de los ejes del plano cartesiano, evidenciando un desconocimiento por parte del $44 \%$ de estudiantes cuando los ejes son llamados como abscisa y ordenada, no logrando relacionar los ejes $\mathbf{X}$ y $\mathbf{Y}$ con sus respectivos nombres. Por lo tanto cada vez que se le solicitaba realizar alguna actividad correspondiente a los ejes y se usaba tal denominación, los estudiantes terminaban intercambiándolos. Caso que se pudo observar en el $52 \%$ de los estudiantes cuando se le preguntó por la posición de la primera y la segunda coordenada del par ordenado en los ejes del plano, debido a que no lo conocen con la denominación usada no lograron indicar la posición. Lo mismo sucede con el $36 \%$ de los estudiantes cuando se le solicita ubicar pares ordenados en el plano cartesiano, al denominar los ejes como $\mathbf{X}$ y $\mathbf{Y}$ respectivamente logran ubicar los pares ordenados de forma correcta, pero cuando se le cambiaba la denominación, automáticamente presentaba dificultades en la ubicación. Al analizar cada una de las pruebas se evidenció inconvenientes en la representación gráfica en el plano cartesiano a partir de una indicación en lenguaje natural, además de un problema de reconocimiento de los ejes, sin embargo, cabe resaltar que el $88 \%$ de los estudiantes interpretan de forma correcta la 
información que se presenta en el plano cartesiano. Finalizando la prueba, se le presenta al estudiante un problema contextualizado para introducirlo al concepto de función presentando el enunciado en lenguaje cotidiano, se le solicita pasarlo al registro tabular obteniendo que el $84 \%$ de estudiantes hacen un proceso correcto, pero solo el $48 \%$ logra darle sentido al problema y trabajar los datos de la forma como este se lo indica y proponiendo una respuesta a la pregunta generada en el contexto del enunciado.

\section{Segundo Momento: Intervención Pedagógica}

Con base en lo obtenido en el análisis de la prueba inicial se elaboran las actividades con las cuales se trabajaron durante siete sesiones de 120 minutos cada una, pero agrupadas en tres grandes secciones temáticas. Cada actividad está enfocada en fortalecer el concepto de función a partir de la articulación de diferentes registros en los que se pueden representar.

En cada sesión se les presenta la actividad a realizar y se les da un tiempo de 90 minutos para resolver las situaciones presentadas en grupos de tres estudiantes cada uno. Cabe resaltar que en cada sesión se hace énfasis en un tipo de representación y a partir de ella se le pide al estudiante hacer transformaciones coherentes. De igual forma se trabajan conceptos básicos y el reconocimiento de los diferentes tipos de funciones. A continuación se hace una descripción de los aspectos más destacados en cada una de las secciones en que se dividió la temática.

Sección 1. En la primera sección se decide trabajar sobre la definición de función, partiendo con la pregunta "Para usted y de acuerdo a lo estudiado en el colegio ¿qué es función?" Al realizar esta pregunta inicialmente las respuestas de los docentes en formación permiten en un punto de referencia para la solución acertada y coherente de los demás ítems. La dificultad observada en los integrantes de la muestra en este primer ítem es que la mayoría replicaron la definición que dieron cuatro o cinco estudiantes una función es una relación donde cada elemento del conjunto de partida se asocia con solo un elemento del conjunto de llegada. Sin embargo en algunos casos los estudiantes dejaban en blanco o expresaron no saber.

El segundo ítem de la actividad 1 consiste en que a partir de la respuesta a ¿qué es función? los docentes en formación analicen varios enunciados y generen una expresión algebraica además de verificar si el enunciado corresponde o no a una función. Para este ítem se hace necesario que los estudiantes tengan conocimiento del concepto de función de lo contrario no podrán determinar si el enunciado representa una función. Al analizar las respuestas, se pudo evidenciar que en el momento de generar la expresión algebraica presentaban inconvenientes para distinguir la variable dependiente e independiente. Además para decidir si era o no función, los docentes en formación hacen un proceso mecánico que consiste en tabular y graficar, de esta forma determinar si es función.

En las siguientes situaciones se busca que los estudiantes reconozcan una función a partir del registro gráfico para luego proponer su respectiva expresión algebraica. Cuando analizan las gráficas, utilizan el criterio de la línea vertical para verificar si corresponde a una función y para hallar la expresión algebraica la mayoría identifican dos puntos y a partir de la ecuación pendiente y puntopendiente la determinan.

Sección 2. En la segunda sección se trabajó representación de funciones a partir del registro gráfico. En la primera situación planteada se busca que los estudiantes relacionen el tipo de función con su representación gráfica y de esta forma verificar si los estudiantes reconocen los tipos de funciones. En el segundo ítem se trabaja el concepto de función inyectiva, sobreyectiva o biyectiva. Se les entrega la expresión algebraica y a partir de ella generan la gráfica con la cual determinan si la función es inyectiva, sobreyectiva o biyectiva. Siendo conceptos que se trabajan desde el colegio se espera que los estudiantes los puedan identificar sin ningún inconveniente; sin embargo los estudiantes expresan no conocer o no 
haber visto esta temática en el colegio, afirmaban que era la primera vez que escuchaban esos términos por lo que la mayoría no respondieron el ítem y decidieron apoyarse en compañeros que sí entendían para que les explicara haciendo uso del trabajo cooperativo.

Las siguientes situaciones se establecieron para el análisis de gráficas determinando los intervalos de crecimiento y decrecimiento de las funciones presentadas y también la interpretación de las gráficas dando una descripción del comportamiento de la función. En este ítem los docentes en formación fueron muy acertados al determinar los intervalos y en la interpretación de las gráficas dando un aporte argumentativo del comportamiento de la gráfica.

Sección 3. La tercera sección se trabajó del registro del lenguaje natural al algebraico y fue donde mayor dificultad se encontró. En toda la actividad se trabajaron enunciados con los cuales los docentes en formación debían hacer la respectiva transformación al registro algebraico, sin embargo presentan dificultades en la interpretación y por ende no son capaces de generar la expresión algebraica. A diferencia de cuando se les daba el registro algebraico y se les pedía hacer una interpretación y generar un enunciado. Los integrantes de la muestra tienen una gran dificultad de análisis y comprensión de textos, presentando mayor inconvenientes cuando los enunciados hacen referencia a una función a tramos.

Durante las intervenciones que se han realizado se ha evidenciado que los docentes en formación aplican el trabajo cooperativo debido que a medida que van teniendo dudas acerca de algún ítem debatían entre ellos y llegaban a una conclusión. De igual forma se ha evidenciado que presentan inconvenientes cuando se les pide analizar un enunciado y verificar si es función o no, dificultando la articulación de los diversos registros de representación.

Cabe resaltar que al finalizar el tiempo de la solución de actividades se realiza la plenaria de
30 minutos donde se socializan aquellos ítems que presentaron mayor diversidad de respuestas de forma que ellos compartían lo que hicieron argumentando su proceder, hasta llegar a una puesta en común entre todos los grupos.

\section{Tercer Momento: Prueba Final}

En el momento que se finalice las sesiones establecidas y se haga el análisis general de las actividades desarrolladas se elaborará la prueba final, enfocándose principalmente en el reconocimiento de funciones en cualquier tipo de representación y de igual forma por medio de ella se buscará identificar si lo encontrado en la prueba inicial ha cambiado en el transcurso de la intervención pedagógica.

La prueba final permitirá dar cuenta de los efectos que se dieron con la aplicación de secuencias didácticas en torno a las dificultades presentadas por los docentes en formación, alrededor del concepto de función. La prueba cuenta con un total de 5 ítem, donde se podrá medir la articulación coherente de los diversos registros de representación, se inicia mostrando a los estudiantes diferentes gráficas, donde se debe identificar si representan funciones y en caso afirmativo, deberán determinar el dominio y rango. Esto permitirá analizar si los docentes en formación logran articular las representaciones gráficas con las algebraicas. Los siguientes cuatros ítems se enfocan en el análisis y transformación de los registros algebraico-gráfico. gráfico-algebraico. Se espera que lo docentes en formación logren hacer la articulación de los diferentes registros de representación de una función.

\section{Conclusiones}

Siguiendo las características de la metodología usada en la investigación, cabe resaltar que se han desarrollado tres de las cuatro etapas propuestas:
1. Análisis preliminar
2. Concepción y análisis a priori
3. Experimentación 
Donde se ha podido evidenciar habilidades $\mathrm{y}$ concepciones de los estudiantes alrededor del concepto de función. Una de las habilidades más frecuente en los estudiantes es el proceso mecanicista para determinar la gráfica de una función, derivada de la educación tradicional del concepto que los docentes en formación traen desde la educación básica media. De igual forma se evidencian vacíos, el desconocimiento de conceptos como función inyectiva, sobreyectiva además de no reconocer funciones a trozos en ningún tipo de registro. Uno de los inconvenientes más comunes en los docentes en formación fue la interpretación y análisis de enunciados, presentan dificultades de comprensión.

A partir de lo analizado de las secuencias aplicadas hasta el momento se espera que al culminar el proceso se vea un efecto de cambio en las dificultades presentadas por parte de los estudiantes y en el momento de la aplicación de la prueba final puedan desarrollar las actividades sin inconvenientes. Es necesario resaltar que este es un proceso de mejoramiento continuo por lo que las secuencias de intervención pedagógica se irán modificando en sus actividades con el fin de obtener mejores resultados en pruebas futuras. Uno de los cambios ya establecidos es implementar las funciones a trozos y la interpretación de enunciados en la mayoría de sesiones para reforzar esta problemática que es la que más persiste en los docentes en formación.

\section{Referencias}

Eisenberg, T. (1991). Functions and associated learning difficulties. InAdvanced mathematical thinking (pp. 140-152). Springer Netherlands.

Hitt, F. (1998). Difficulties in the articulation of different representations linked to the concept of function. The Journal of Mathematical Behavior, 17(1), 123-134.

Lopéz, J., Sosa, L. (2008) Dificultades conceptuales y procedimentales en el aprendizaje de funciones en estudiantes de bachillerato. 308-318. Recuperado de: http://funes.uniandes.edu.co/4946/1/ L\%C3\%B3pezDificultadesALME2008.pdf

Prada-Núñez, R., Hernández-Suárez, C., \& Ramírez-Leal, P. (2016). Comprensión de la noción de función y la articulación de los registros semióticos que la representan entre estudiantes que ingresan a un programa de Ingeniería. Revista Científica, 2(25), 188-205.

Tobón Sergio. Pimienta Julio, H. García Juan, A. (2010). Secuencias didácticas: aprendizaje y evaluación de competencias (1a ed.). México: Grupo Editorial Pearson. 